\title{
Surgical treatment of arterial lower-limb injuries by fire arms
}

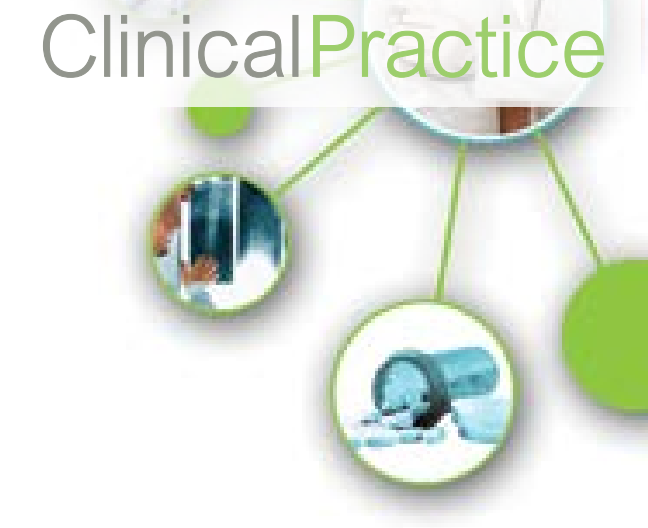

Background: Arterial ballistic injuries of the limbs are rarely encountered in civilian practice and the protocols of care are generally based on the experience gained during military conflicts. The aim of this study is to describe the medicosurgical management of the ballistic injuries of lower limbs with arterial damage and to develop a strategy for this type of rare lesions.

Patients and Methods: It is a retrospective study of 11 male patients who underwent surgery for vascular lesions in the extremities caused by firearms.

Results: The mean age was 33 years. The initial presentation in the emergency room was a hemodynamic shock in 5 patients, acute ischemia in 9 patients; externalized bleeding in 6 cases and one patient was seen at the aneurysm complication stage. Superficial femoral artery and popliteal artery were the most affected. Eight patients had also venous lesions and four patients had bone and nerve damage. All patients underwent arterial restoration by venous bypass surgery in $83.4 \%$ of cases and by end-to-end anastomosis in $16.6 \%$; simultaneously, $50 \%$ of the venous lesions and $75 \%$ of the nerve lesions were repaired. Two patients were dead, one for bleeding shock and one for septic shock. The mean follow-up was 18 months. Three patients had surgical site infection and one patient had graft thrombosis.

Conclusion: The management of vascular injuries caused by firearm requires surgical treatment by a multidisciplinary team with good results in the medium and long term essentially with isolated vascular injuries. Postoperative mortality and morbidity are directly related to hemodynamic status, surgical repair of vascular injuries and associated lesions in time, which leading to clear and rapid management.

Keywords: Trauma, artery, gunshot, surgery.

\section{Introduction}

Ballistic Vascular injuries were generally encountered during wars. Armed conflicts have contributed significantly to the evolution of vascular surgery in general and to the development of many strategies for the management of ballistic injuries in particular especially in the United States [1]. Firearm injuries were rare in Tunisia until the outbreak of the revolts of the Arab countries. We report our series of 11 patients operated for vascular trauma by firearms at the lower limbs.

\section{Patients and Methods}

This is a retrospective and descriptive study including 11 patients operated for lower limb firearm injuries involving vascular lesions. The purpose of the study is to evaluate medicosurgical management, analyze the severity of the ballistic trauma and the various complications and to develop a therapeutic strategy for this type of lesions.

\section{Results}

The average age was 33 years [15-46 years]. Five patients presented with hemorrhagic shock and directly putted under vasoactive drugs. All patients had unilateral vascular lesions. Pain and coldness were observed in 10 patients, paresthesia in 7 and paralysis in 2 patients. At admission, $50 \%$ of patients had active externalized bleeding. All the patients had pulse abolition downstream of the lesions except one who had an incomplete lesion of the popliteal artery. Peri-operative blood transfusion was required in seven patients, with an average of 4 globular pellet pockets.

According to the physical examination and
Bilel Derbel, Jalel Ziadi, Sobhi Mleyhi*, Rim Miri, Malek Ben Mrad, Faker Ghédira, Raouf Denguir

Department of Cardiovascular surgery, la Rabta Hospital,Medical institute of Tunis. Tunis EL MANAR University, Tunisia

\section{*Author for correspondence:}

mleyhisobhi@yahoo.com 
standard radiographs, armored bullet traces were found in seven patients (FIGURE 1) and lead bullets in three patients (poly-splitting injury (FIGURE 2). In 50\% of cases, an inlet and an outlet have been identified (FIGURE 3); with an average diameter of $1.2 \mathrm{~cm}$ and 5 $\mathrm{cm}$ respectively. One patient has been seen at the stage of false aneurysm complication. The superficial femoral artery is the most frequently affected ( $43 \%$ of cases). A venous lesion was associated with arterial damage in 8 cases, a bone lesion in 5 cases and a nerve injury in 4 cases.

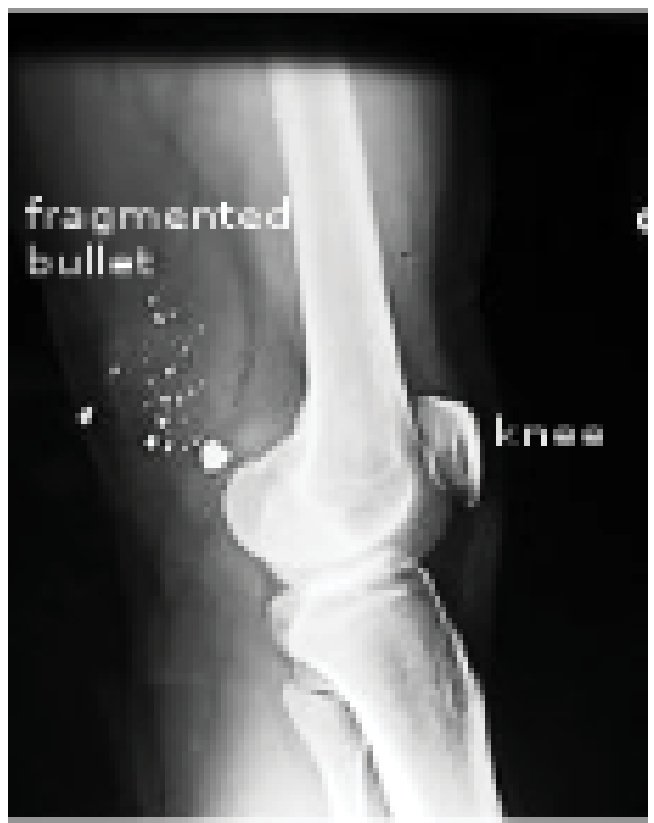

Figure 1: Knee X-ray shown jackted bullet fragmented.

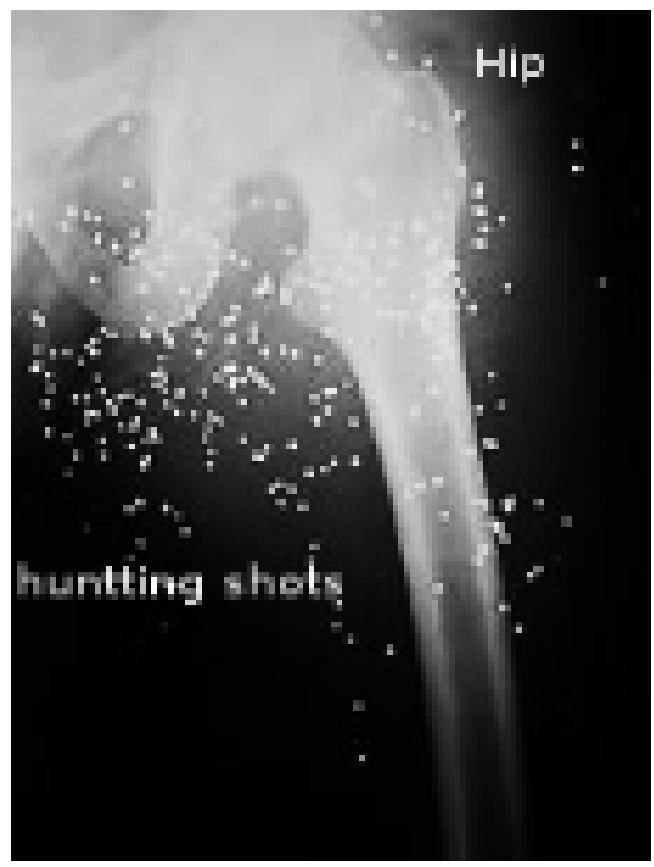

Figure 2: Front hip X-ray shown buckshot.
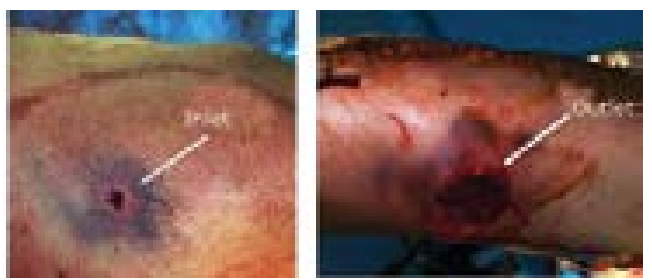

Figure 3: Bullet inlets and outlets.

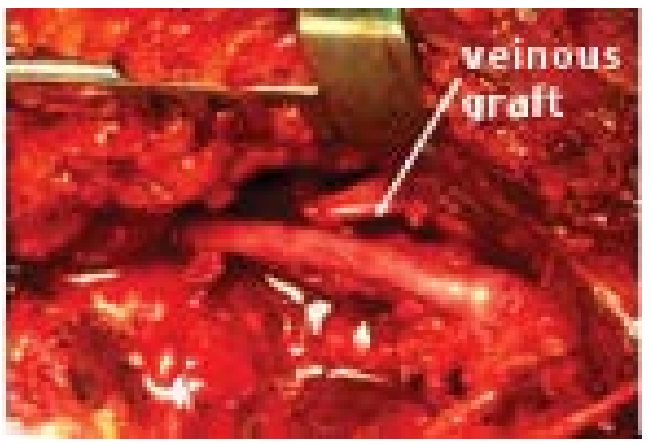

Figure 4: Per-operative image of venous popliteo-popliteal graft.

Arterial revascularization was performed urgently for 10 patients with an average delay of 8 hours [2 - 33 hours] and only one patient was treated after 5 months of the accident at the stage of aneurysmal complication. Ten grafts were performed by internal saphenous graft (FIGURE 4) and an end-to-end anastomosis for one patient. Associated venous lesions were repaired by patch in 3 cases and by direct in 2 cases; while injured distal veins were ligated. The four patients with bone fractures underwent surgical revascularization after fracture reduction. Three of the four patients with nerve injuries underwent surgical treatment (neurolysis or nerve graft) with specialized rehabilitation. The average hospital stay was 11.6 days with extremes ranging from 2 to 40 days. Two deaths were reported: one by hemorrhagic shock and the second by septic shock with multi-visceral failure. In both cases, the patients were initially operated in other hospitals and were transferred late. The postoperative course was marked by a thrombosis of the bypass in one patient (venous bypass between the subarticular popliteal artery and the posterior tibial artery) and four patients presented an infection of the operating site. Two patients required a skin graft in the third postoperative month. All surviving patients had peripheral pulses 2 years after confirmed by an arterial Doppler.

\section{Discussion}

The incidence of limb trauma by firearms associated with arterial injury is difficult to 
assess and differs from country to country. There are many series in the literature dealing with these traumas; the majority of these series are American [2]. These vascular injuries are less common in the civilian population and rare in our countries until the outbreak of the revolts of the Arab countries. In Tunisia, during the events from December 17, 2010 to January 25, 2011, the hospital structures were faced with a very large flow of victims. The official figures of the "National Fact Finding Commission" report 2174 serious injuries and 338 deaths [3].

The ballistic examination of the wound involves the description of three basic elements: the inlet, the outlet and the path. These elements vary according to the weapon, the ammunition, the distance of fire but especially according to the crossed Tissues [4]. The damage occurred by the projectile energy is explained by crushing, laceration and stretching phenomena.

Pre-hospital care remains a challenge. Stopping bleeding and rapid transportation of the wounded to a specialized center is the cornerstone of this phase.

The femoral artery is the most exposed. Its damage in the Scarpa, especially in the case of a gunshot wound that destroyed the joint, makes immediate haemostasis difficult [5].

The urgency of vascular ballistic trauma, in the presence of shock, can lead to massive arterial hemorrhage, major compressive hematoma or acute limb ischemia; so the patient should be immediately operated without any further exploration [6]. In the other cases and to limit the transition to surgery, doppler ultrasound with measurement of the systolic pressure index as in the Knudson study [7] or angioscan whose indications differ according to the teams are limited to trauma with minor signs [8]. In our series only one patient had an angioscan for false aneurysm 5 months after gone shot injury.

The goal of treatment in ballistic trauma has long been to preserve the life-threatening condition and stop bleeding by simple ligation of the arteries with a high rate of amputation. Since the Korean War, the goal and the means have changed. The goal is to save the patient life but also his limb through vascular restoration whenever possible.

The primary prevention of primary infection remains complete excision of the wound with leaving the wound open for a delayed closure. Antibiotics are just adjunctive therapy to good surgical practice. Administration of heparin (100 $\mathrm{U} / \mathrm{kg}$ ) reduces amputation rates. It prevents thrombosis in the micro-circulation [9].

The rules of vascular repair are not specific to ballistic traumas, they are valid for all limb trauma. The suture should be made in normal tissue, performed without tension or torsion and limb in extension. The choice of the technique depends on the type and extent of the trauma:

Simple suture for partial or total open wounds if the size of the artery allows it without stenosing it, otherwise a venous patch should be used.

Direct end-to-end suture for short or oblique wounds, sub-adventitial rupture or extensive intimal lesions. The termino-terminal suture is performed only if the vessel extremities could be faced without tension.

The interposition of a graft is the most used if the loss of tissue is important. The best material is the venous auto-graft: the saphenous vein is usually used. Prosthetic grafts represent an alternative in case of inadequate or unusable veins despite the known risk of infection. Anatomical revascularization is the best technique. Extraanatomical bypass is preferable in trauma associated with significant tissue contusion with a lack of muscle and skin recovery.

The place of endovascular treatment remains limited and can only be used very selectively. It may be indicated in hemodynamically stable patients without active hemorrhage, in proximal segmental lesions of the limbs such as superficial femoral [10] and axillo-subclavian arterial lesions and in some cases arteriovenous fistulas or false proximal aneurysm. Endovascular techniques also allow embolization in cases of progressive haemorrhagic lesions with difficult or dangerous access.

Despite intimate anatomical relationships between the arteries and their homologous veins, venous lesions during limb trauma are less common than arterial lesions. Hafez et al., [11] in a series of 550 patients with 641 arterial lesions in the lower limbs, report only 144 venous lesions (22.4\%). Some recommend concomitant repair of venous lesions as this helps to maintain the patency of arterial restorations and to prevent postoperative edema [12]. Data from the literature supports venous repair, which is more selective, depending on the location of the lesion. Indeed, several authors indicate the repair of proximal venous 
lesions [13,14]. Nevertheless, the benefit of this repair is technical dependent. Techniques other than lateral sutures and termino-terminal anastomoses, such as patch repair or end-vein repair, require a long operating time with an uncertain result. In addition, venous repairs are associated with a high probability of deep vein thrombosis [10]. The failure of its repairs is also associated with a high rate of pulmonary embolism (22\%) [15]. For these reasons, treatment with unfractionated heparin followed by oral anticoagulation for at least 3 months is recommended in the case of venous restoration [10].

The decision of immediate limb amputation remains difficult and must be taken by all the surgical teams (orthopedist, plastic and vascular surgeons, anesthesist).

Early mortality in war-related limb trauma is mainly related to massive external bleeding, too long evacuation delay, late resuscitation and the occurrence of infection [16]. Its incidence varies from $1.3 \%$ to $13 \%[17,18]$. Morbidity is mainly represented by the occurrence of early bypass thrombosis, postoperative bleeding and infection [19]. Prevention is an essential element in care; it is based essentially on early antibiotic prophylaxis and careful wound dressing, as well as tetanus prevention.

\section{Conclusion}

Lower Limbs ballistic vascular injuries are usually encountered during military conflicts. They are less frequent in the civilian population with very different incidences from one country to another. These traumatisms are most often responsible for complex lesions, putting at risk the vital and functional prognosis. Management must be rapid and multidisciplinary in a specialized center to minimize short-term morbidity and mortality and improve functional outcomes in the medium and long term. 


\section{REFERENCES}

Hull JB. Management of gunshot fractures of the extremities. J. Trauma Acute Care Surg. 40(3S), 193S-197S (1996).

Mäkitie I, Mattila VM, Pihlajamäki H. Severe vascular gunshot injuries of the extremities: a ten-year nation-wide analysis from Finland. Scand. J. Surg. 95(1), 49-54 (2006).

La commission nationale d'établissement des faits. Le rapport final de la commission nationale d'investigation. Tunis: Conférence de presse. (2012).

Pons J. Mécanisme des lésions par balle. Def. Arm. 50, 49-52 (1986).

Mattox KL, Feliciano DV, Burch J, Beall AC Jr, Jordan GL Jr, De Bakey ME. Five thousand seven hundred sixty cardiovascular injuries in 4459 patients. Epidemiologic evolution 1958 to 1987. Ann. Surg. 209(6), 698-707 (1989).

Smith RF, Elliot JP, Hageman JH, Szilagyi DE, Xavier AO. Acute penetrating arterial injuries of the neck and limbs. Arch. Surg. 109(2), 198-205 (1974).

Knudson MM, Lewis FR, Atkinson
K, Neuhaus A. The role of duplex ultrasound arterial imaging in patients with penetrating extremity trauma. Arch. Surg. 128(9), 1033-1038 (1993).

Hoyt DB, Coimbra R. Initial care, operative care and postoperative care. In Vascular trauma (2nd ed). Elsevier Science. 97-112 (2004).

Guerrero A, Gibson K, Kralovich KA et al. Limb loss following lower extremity arterial trauma: what can be done proactively? Injury 33(9), 765-769 (2002).

Franz RW, Shah KJ, Halaharvi D, Franz ET, Hartman JF, Wright ML. A 5-year review of management of lower extremity arterial injuries at an urban level I trauma center. J. Vasc. Surg. 53(6), 160410 (2011).

Hafez HM, Woolgar J, Robbs JV. Lower extremity arterial injury: results of 550 cases and review of risk factors associated with limb loss. J Vasc Surg. 33(6), 1212-1219 (2001).

Timberlake GA, Kerstein MD. Venous injury: to repair or ligate, the dilemma revisited. Am. Surg. 61(2), 139145 (1995).

Phifer T, Gerlock A, Vekovius W, Rich
$\mathrm{N}$, McDonald J. Amputation risk factors in concomitant superficial femoral artery and vein injuries. Ann. Surg. 199(2), 241243 (1984).

Gorman J F. Combat wounds of the popliteal artery. Ann. Surg. 168, 974-980 (1968).

Cargile JS, Hunt JL, Purdue GF. Acute trauma of the femoral artery and vein. $J$. Trauma. 32(3), 364-370 (1992).

Nitecki SS, Karram T, Ofer A, Engel A, Hoffman A. Management of combat vascular injuries using modern imaging: are we getting better? Emerg. Med. Int. 2013, 689473 (2013).

Hughes CW. Acute vascular trauma in Korean War casualties; an analysis of 180 cases. Surg. Gynecol. Obstet. 99(1), 91-100 (1954).

Spencer FC, Grewe RV. The management of arterial injuries in battle casualties. Ann. Surg. 141, 304-313 (1955).

Brown KV, Ramasamy A, Tai N, et al. Complications of extremity vascular injuries in conflict. J. Trauma. 66, S145149 (2009). 